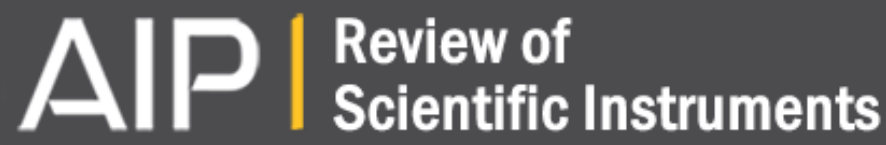

\section{Novel schemes for the optimization of the SPARC narrow band THz source}

B. Marchetti, A. Bacci, E. Chiadroni, A. Cianchi, M. Ferrario, A. Mostacci, R. Pompili, C. Ronsivalle, B. Spataro, and I. Zagorodnov

Citation: Review of Scientific Instruments 86, 073301 (2015); doi: 10.1063/1.4922882

View online: $\mathrm{http}: / / d x$. doi.org/10.1063/1.4922882

View Table of Contents: http://scitation.aip.org/content/aip/journal/rsi/86/7?ver=pdfcov

Published by the AIP Publishing

\section{Articles you may be interested in}

High-field half-cycle terahertz radiation from relativistic laser interaction with thin solid targets Appl. Phys. Lett. 103, 204107 (2013); 10.1063/1.4831684

Terahertz radiation source based on self-wake beam bunching AIP Conf. Proc. 1507, 421 (2012); 10.1063/1.4773733

Single-cycle terahertz pulses with $>0.2 \mathrm{~V} / \mathrm{A}$ field amplitudes via coherent transition radiation Appl. Phys. Lett. 99, 141117 (2011); 10.1063/1.3646399

High-precision frequency measurements in the $\mathrm{THz}$ spectral region using an unstabilized femtosecond laser Appl. Phys. Lett. 99, 121111 (2011); 10.1063/1.3640234

Novel THz Radiation Sources

AIP Conf. Proc. 807, 390 (2006); 10.1063/1.2158803

Frustrated by

old technology?

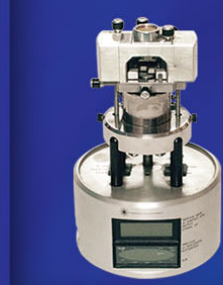

Is your AFM dead

and can't be repaired?

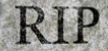

My Old AFM $1994-2015$
Sick of bad customer support?

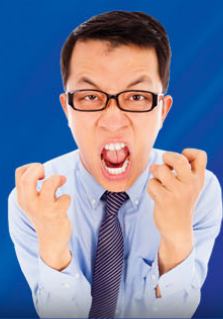

It is time to upgrade your AFM Minimum \$20,000 trade-in discount for purchases before August 31st

Asylum Research is today's technology leader in AFM 


\title{
Novel schemes for the optimization of the SPARC narrow band THz source
}

\author{
B. Marchetti, ${ }^{1, \text { a) }}$ A. Bacci, ${ }^{2}$ E. Chiadroni, ${ }^{3}$ A. Cianchi, ${ }^{4,5}$ M. Ferrario, ${ }^{3}$ A. Mostacci, ${ }^{6,7}$ \\ R. Pompili, ${ }^{3}$ C. Ronsivalle, ${ }^{8}$ B. Spataro, ${ }^{3}$ and I. Zagorodnov ${ }^{1}$ \\ ${ }^{1}$ DESY, Hamburg, Germany \\ ${ }^{2}$ INFN MI, Milan, Italy \\ ${ }^{3} I N F N-L N F$, Frascati, Italy \\ 4"Tor Vergata" University of Rome, Rome, Italy \\ ${ }^{5}$ INFN Roma "Tor Vergata," Rome, Italy \\ 6 "Sapienza" University of Rome, Rome, Italy \\ ${ }^{7}$ INFN Roma, Rome, Italy \\ ${ }^{8}$ ENEA C.R., Frascati, Italy
}

(Received 12 February 2015; accepted 8 June 2015; published online 1 July 2015)

\begin{abstract}
A pulsed, tunable, narrow band radiation source with frequency in the $\mathrm{THz}$ region can be obtained collecting the coherent transition radiation produced by a train of ultra-short electron bunches having picosecond scale inter-distance. In this paper, we review the techniques feasible at the SPARC_LAB test facility to produce and manipulate the requested train of electron bunches and we examine the dynamics of their acceleration and compression. In addition, we show how the performances of the train compression and the radiation intensity and bandwidth can be significantly improved through the insertion of a fourth order harmonic cavity, working in the X-band and acting as a longitudinal phase space linearizer. (C) 2015 AIP Publishing LLC. [http://dx.doi.org/10.1063/1.4922882]
\end{abstract}

\section{INTRODUCTION}

The generation and manipulation of a train of electron bunches, also called comb beam, with sub-picosecond length and adjustable (sub)-picosecond inter-distance is itself challenging and very interesting due to its wide spectrum of applications, such as intense narrow band $\mathrm{THz}$ radiation, ${ }^{1}$ ultrafast pump-probe Free Electron Laser (FEL) experiments, ${ }^{2-5}$ and coherent excitation of plasma waves in plasma accelerators. ${ }^{6}$

In the last decades, many methods to produce a train of electron bunches have been investigated.

Passive techniques realize cuts in the beam distribution in order to shape the beam charge profile. These cuts can be applied in several ways. By placing a mask in a high dispersion, low beta function region of a dogleg along the beam line, it is possible to produce a well defined temporal bunch train out of a long bunch with correlated energy spread. ${ }^{7}$ Alternatively, a transversely segmented beam can be generated with a multislit mask via a transverse-to-longitudinal phase space exchange technique, obtained by placing a deflecting cavity between two dispersive sections. ${ }^{8}$ The passive techniques foresee charge losses; therefore, the maximum charge per sub-bunch is typically few tens of $\mathrm{pC}$ and the lost charge heats up the elements of the beamline where the slit is placed. The corresponding radiation increase is proportional to the repetition rate of the accelerator and might become a limit for the allowed running time of the machine. Moreover, the possibility to continuously tune the spacing between the pulses is desirable.

Active techniques, on the other side, manipulate the beam charge distribution and do not foresee any loss of charge. Resonant Cherenkov radiators are promising for the realization of user-customized (i.e. non-frequency tunable) compact

\footnotetext{
a)barbara.marchetti@desy.de
}

radiation sources. ${ }^{9}{ }^{10}$ In this case, the modulation of the subbunches is simply impressed by a RF buncher. The so-called bunch slicing technique allows to obtain only a modulation on the single bunch strong enough to modify the spectrum of the emission and produce $\mathrm{THz}$ radiation. ${ }^{11,12}$ The frequency of the modulation is given by the seeding laser in use and the number of particles responsible for the coherent emission is only a small fraction of the entire electron bunch. Finally, the interaction of a long bunch with its wakes after passing through a dielectric loaded waveguide induces an energy modulation that can be further converted into electron bunches formation. ${ }^{13}$ This method allowed the production of trains of bunches with a spacing tunable in the range $0.68-0.9 \mathrm{THz}$ and is promising for the realization of table top sources tunable within a small frequency range.

In this scientific environment, at the SPARC_LAB test facility ${ }^{14}$ for the first time, RF compression has been applied to an electron beam produced by a longitudinally modulated laser pulse, the so-called comb pulse. In the SPARC_LAB case, the production of the bunch train does not rely on beam losses, thus it is suitable for the production of higher charge bunches. ${ }^{15}$ In addition, the final bunch train parameters can be tuned by playing with the laser pulse shaping and varying the accelerator working conditions. ${ }^{16}$ Compared with the other methods previously cited, this scheme is particularly flexible in terms of tunability of the emission frequency and tunability of the charge contained in each sub-bunch of the train. The latter characteristic is especially important for the application in resonant beam driven plasma wakefield accelerators. ${ }^{7}$

In Ref. 17, the laser comb scheme has been first theoretically discussed. The train of electron bunches can be produced illuminating a metallic photo-cathode with a comb-like laser pulse. In this configuration, the electrons in each sub-pulse experience a large longitudinal space charge field with a linear 
correlation along the sub-bunch. The work done by the space charge force produces an energy modulation within the subpulse that can be transformed into a density modulation by a compressor (magnetic or RF).

The manipulation under RF compression of a train of electron bunches, consisting of two ${ }^{18}$ or four pulses, has been described in Ref. 16 together with the first experimental results obtained at $S P A R C_{-} L A B$. It has been experimentally demonstrated that it is possible to control the pulse spacing, length, current, and energy separation by properly setting the accelerator.

The compression of the comb beam is very interesting from the beam dynamic point of view: two compressions take indeed place in parallel. First of all, there is the compression of the train that is used to tune the spacing between the subbunches and is mainly regulated by the phases and amplitudes of the RF cavities. Second, we observe the compression of each single sub-bunch, which is allowed by the chirp impressed by the space-charge force and is strongly affected by the photo-cathode laser spot-size and the transverse focusing of the beam. In Ref. 16, it has been shown that the optimal working point for the comb compression using the velocity bunching is when the train is deeply overcompressed. In this case, indeed the single sub-bunches are short and they are well separated in time.

When the comb beam impinges the metallic target, an almost monochromatic pulse of radiation centered at the wavelength of the spacing between the micropulses is produced. The emission from an electron bunch with longitudinal charge profile described by the function $g(z)$ is given by ${ }^{19}$

$$
\frac{d^{2} I}{d \omega d \Omega}=\frac{d^{2} I_{s p}}{d \omega d \Omega}[N+N(N-1) F(\omega)],
$$

where $N$ is the total number of particles in the bunch and $F(\omega)$ is the longitudinal form factor of the bunch, defined as

$$
F(\omega)=\left|\int_{-\infty}^{\infty} g(z) e^{-i \frac{\omega}{c} z} d z\right|^{2}
$$

that relates the bunch distribution with the phases of the electrons inside the bunch and $0 \leq F(\omega) \leq 1$. The form factor corresponding to a train of electron bunches shows peaks at the characteristic frequency of the comb structure and its higher harmonics, as shown in Figure 1. The carrier curve of the peaks is given by the form factor of one single sub-bunch; thus, the amplitude of the peaks depends on the length of each subbunch and their bandwidth becomes smaller if the number of the sub-bunches increases. In the present paper, we will use the form factor as figure of merit of the radiation produced by the comb beam at the end of the dogleg.

The main advantage in using train of bunches to produce $\mathrm{THz}$ radiation is that it makes possible to reach short (sub-ps) sub-bunch length together with high total charge by increasing the number of sub-bunches in the train. As Eq. (1) states, the higher is the total charge, the higher is the amplitude of the energy spectrum. It is important to stress that the comb scheme makes in principle possible to have higher total charge by raising the number of sub-bunches without influencing the single pulse length.

On the other side, the main disadvantage of the comb scheme is the difficulty of obtaining a well periodic structure after the bunch compression.

The experimental results of the application of such a scheme for the SPARC_LAB THz source have been published in Ref. 19. Different THz emission regimes have been achieved by a proper control of pulse shaping, length, charge, and energy
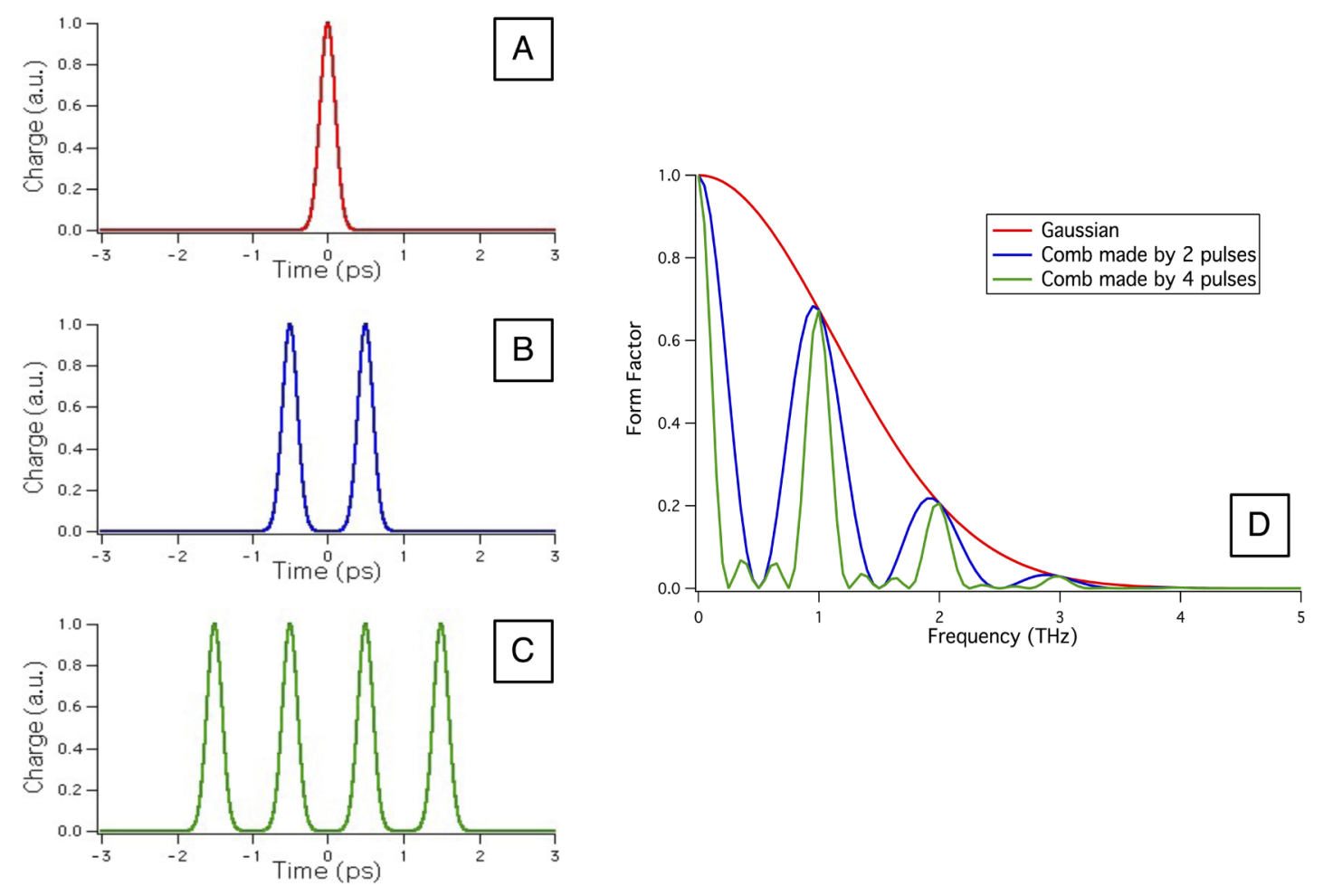

FIG. 1. Plot (d) shows the form factors corresponding to the longitudinal charge profiles (a), (b), and (c) on the left side. 
separation, thanks to a proper set of both the injector and dogleg parameters. In addition to the comb scheme, also the single bunch scheme has been investigated. The latter allows the generation of a broad band $\mathrm{THz}$ energy spectrum. ${ }^{20}$

The present paper focuses the attention on the study of the best method feasible at $S P A R C_{-} L A B$ for the compression of the comb beam. In the SPARC_LAB test facility, indeed both $\mathrm{RF}$ and magnetic compressions are in principle possible and we want to point out which one of the two techniques is more convenient to use for the narrow band $\mathrm{THz}$ source. Our scope is on one side to investigate the range of tunability of the narrow band THz source at SPARC_LAB and clarify the effect of the different machine knobs that can be used to compress the train; on the other side, we want to have a better understanding of the differences between the two techniques and highlight the factors that prevent the achievement of a fully periodic comb beam at the target position. At the end of the discussion, we choose to work with the RF compression technique and we show how the insertion of a fourth order harmonic cavity can significantly improve the quality of the final comb beam.

\section{SIMULATIONS}

At $S P A R C_{-} L A B$, the electron bunch is produced by photoemission from a copper cathode and accelerated up to about $150 \mathrm{MeV}$ at the end of the main linac. In Fig. 2, the layout of the facility is shown. The first linac section is often used as RF compressor. The line in front of the main linac is currently used for Self Amplified Spontaneous Emission (SASE) and seeded FEL radiation production. The second line is a test bench beamline for beam diagnostics studies and $\mathrm{THz}$ radiation experiments. $\mathrm{THz}$ radiation is characterized by measuring, through an interferometer, the spectrum of Coherent Transition Radiation (CTR) emitted by a sub-ps electron beam interacting with a metallic screen. Just before the target, there is some space ready for the installation of a magnetic compressor whose design is already available. The third and fourth beamlines are used together with the multi-

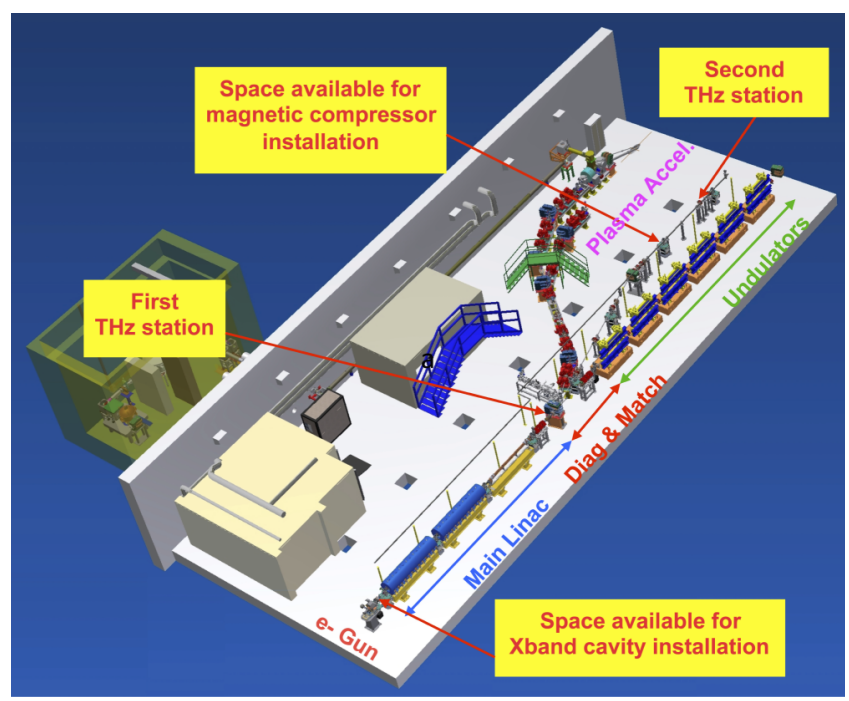

FIG. 2. SPARC LAB layout. hundred TW laser, FLAME: ${ }^{21}$ the third one is dedicated to laser driven plasma acceleration with an externally injected high brightness electron beam, while the fourth one drives a source of gamma rays through Thomson back-scattering. ${ }^{22}$

In this section, we are going to explore two bunch compression schemes plus the introduction of a linearizing cavity in the first of them.

The RF compression scheme is the method already in use at $S P A R C \_L A B$. Experimental results for this scheme are already available and published in Refs. 16, 18, and 19, but we would like to discuss here which parameters of the machine can be varied in order to tune the peaks of the emission frequencies.

The second method explored is the magnetic compression. Figure 2 shows that the second beamline of SPARC_LAB could possibly host a magnetic compressor. We want to investigate if the magnetic compression scheme can provide better results than the velocity bunching in terms of the amplitude of the form factor and tunability of the emission wavelength.

Finally, in Sec. II C, we study a RF compression scheme with the compensation of the non-linear terms in the longitudinal phase space of the beam through the insertion of an $\mathrm{X}$-band cavity after the gun.

In the following, we will always use the form factor as figure of merit to compare the results achievable with the different schemes. We are particularly interested in increasing the local amplitude of the form factor, diminishing the bandwidth of its peaks, and be able to shift their position.

For the simulations presented in this section, we have used different comb beam distributions produced using a laser pulse constituted by four sub-pulses with the same length, relative distance of about 2 ps or 4.3 ps and a total charge of $360 \mathrm{pC}$. The beam production, transport, and compression have been tracked using the well known Tstep code. ${ }^{23}$ In the simulations, the space charge field is always included, while the Coherent Synchrotron Radiation (CSR) in the dogleg and bunch compressor are neglected. The reason why this effect has not been included is that we expect it to mainly influence the beam emittance, that is not a critical parameter for the CTR production.

\section{A. RF compression scheme}

When using the RF compression technique, also called velocity bunching, ${ }^{24,25}$ a low energy electron bunch is injected into a RF module close to the 0 phase of the electric field for which the head of the bunch finds a lower field amplitude than the tail. In this way, the bunch is compressed and accelerated at the same time. In fact, thanks to the different propagation velocities of the RF field and the electrons; the latter slip on the higher electric field amplitude region while traveling in the cavity. At the exit of the cavity, the longitudinal phase space of the electron bunch is rotated. The compression factor can be adjusted by slightly changing the injection phase.

The amplitude of the electric field experienced by the particles can be expressed as

$$
\begin{aligned}
V(\phi)= & V_{0} \sin (\phi) \approx V_{0} \sin \left(\phi_{0}\right)+\Delta \phi V_{0} \cos \phi_{0} \\
& -\frac{1}{2}(\Delta \phi)^{2} V_{0} \sin \phi_{0},
\end{aligned}
$$


where $\phi_{0}$ is the injection phase of the electron bunch with respect to the RF cavity, $V_{0}$ is the amplitude of the sinusoidal field, and $\phi=\phi_{0}+\Delta \phi$. When we compress a train of pulses, the sub-bunches enter the RF cavity with a different phase. Even if the longitudinal phase space distributions of the subbunches at the entrance of the cavity have identical energy chirps, they experience a different field amplitude and end up into different compression points (compare, for example, Figures 3(a) and 3(b)).

In Ref. 16, it has already been discussed that when the overall train has its minimum length, the sub-bunches are overlapped in time and the train-like longitudinal distribution is lost. In order to compress the sub-bunches preserving the longitudinal modulation, it is necessary to work with an overcompressed train like in Fig. 3(b).

The RF-field amplitude has a sinusoidal behavior with respect to the time. This non-linear dependence is not visible within one sub-bunch but becomes non-negligible if we look at the overall train: the distance between two subsequent bunches at the cavity exit is indeed not preserved (again compare Figs. 3(a) and 3(b)). Moreover, the energy difference introduced between them may lead to different behaviors when
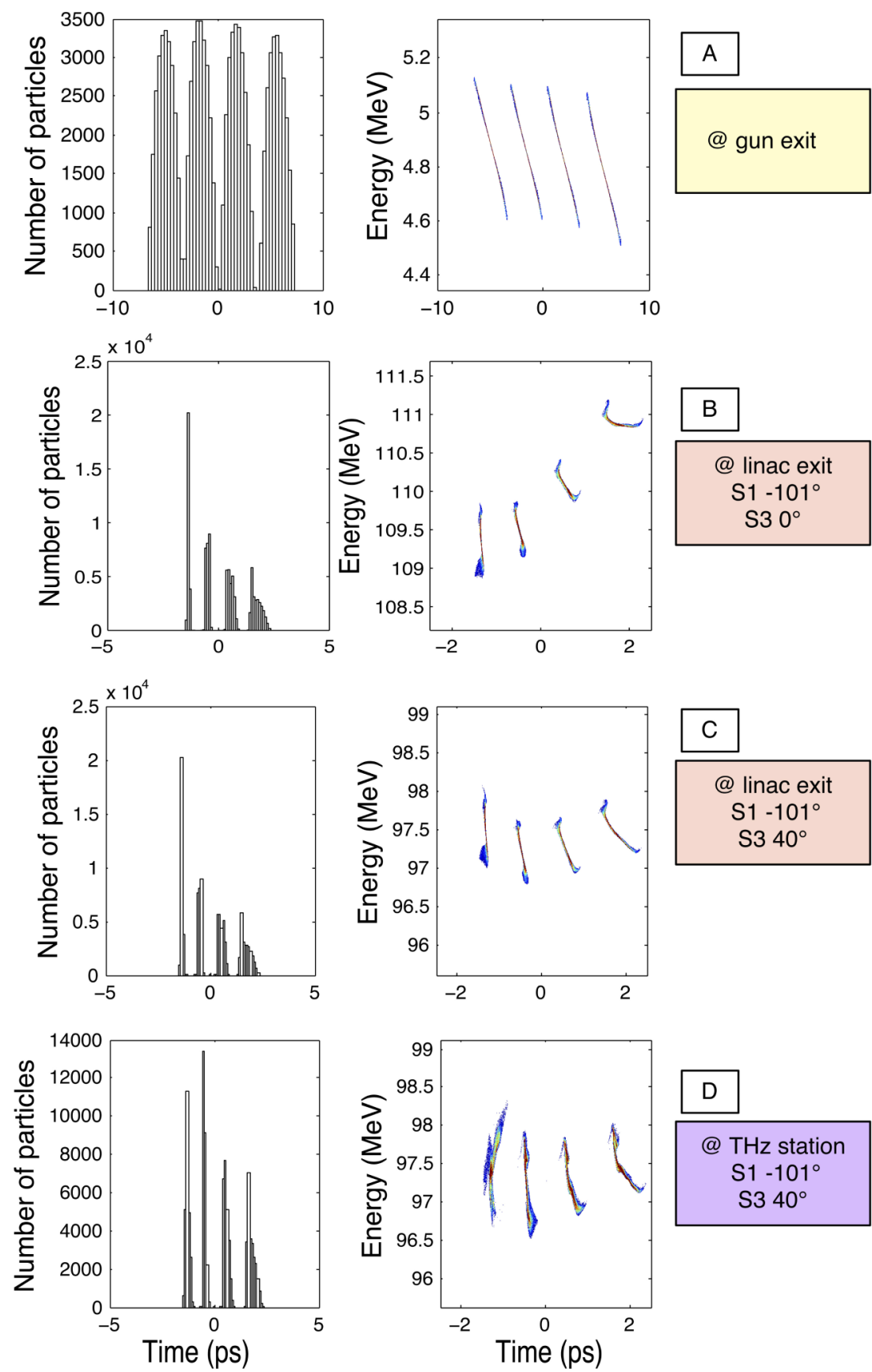

FIG. 3. The compression of a train composed by 4 pulses. (a) Electron bunch charge distribution and longitudinal phase space distribution at the gun exit. (b) Longitudinal charge profile and longitudinal phase space distribution at the exit of the linac. The first accelerating section S1 has been used as RF compressor, while the other two RF sections, named S2 and S3, have been operated on crest. (c) Similar to plot (b) but the third accelerating section S3 has been operated off crest to compensate the energy spread within the electron bunch train. (d) Electron bunch charge distribution and longitudinal phase space at the second THz station. During the transport through the dogleg, the train has been further compressed by the non-zero longitudinal dispersion. ${ }^{26}$ 

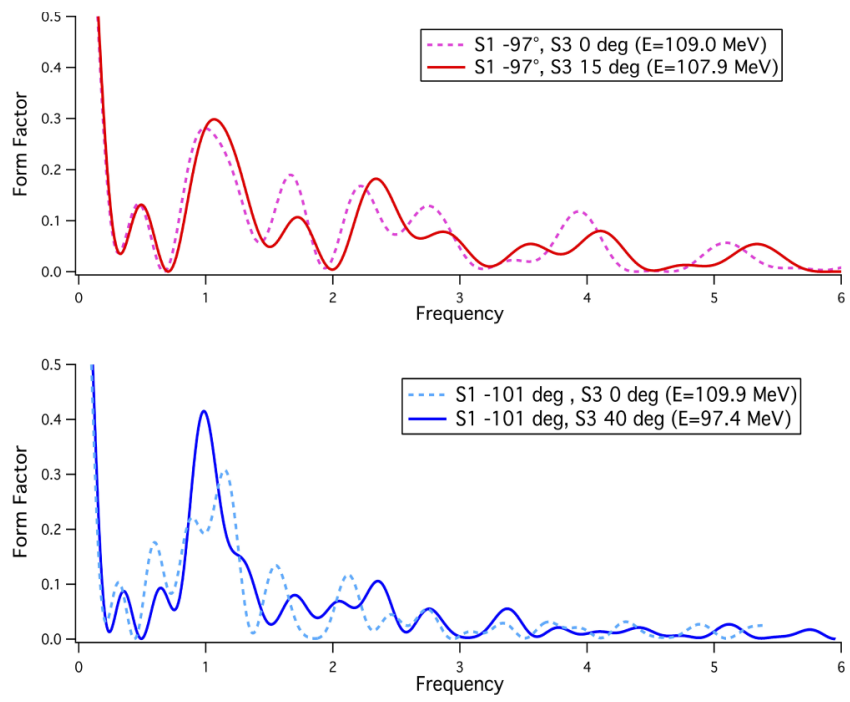

FIG. 4. Form factors at the second $\mathrm{THz}$ station corresponding to different setups of the linac.

traveling through the dogleg. This last effect can be easily compensated by setting the third linac section off-crest (see Fig. 3(c)).

The form factor corresponding to the distribution in Figures 3(c) and 3(d) is presented in Fig. 4. Peaks at about $1 \mathrm{THz}$ and $2.2 \mathrm{THz}$ are visible, which are the frequencies corresponding to the first and second harmonics of the train.

As mentioned in the Introduction, one of the advantages of the use of the comb scheme for $\mathrm{THz}$ radiation production is that the wavelength corresponding to the narrow band peak in the energy spectrum is tunable.

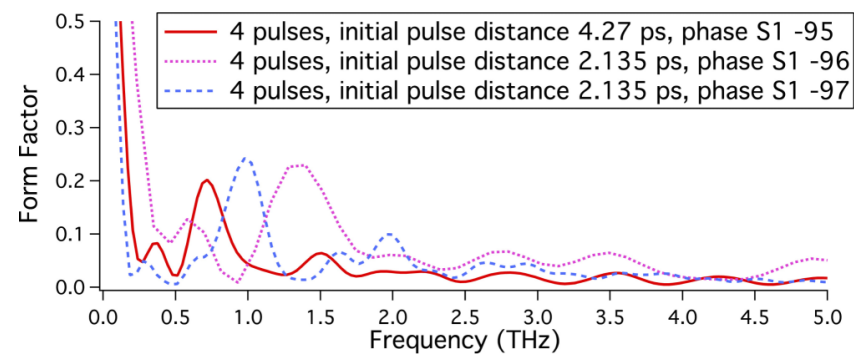

FIG. 5. Form factors corresponding to different compression setups of a four-pulses comb beam. The total charge of the extracted electron beam is $360 \mathrm{pC}$. By varying the spacing of the photo-cathode laser pulses and the phase of the first accelerating cavity, here named S1 and used as a RF compressor, it is possible to tune the final distance between the pulses and thus the coherent emission frequencies.

In Ref. 27, it has been presented the study of the compression of a 2-pulses train that shows how it is possible to change the position of the peaks by changing the RF-gun phase and the phase of the RF compressor. In Fig. 5, the study has been repeated for a comb constituted by 4 bunches. In this second case, the spacing of the pulses of the photo-cathode laser has been used as an additional knob.

\section{B. Magnetic compression scheme}

When an energy chirped beam is injected into a magnetic chicane, particles travel through different paths according to their energy.

The longitudinal dispersion $\eta$ provides the dependence of the longitudinal particle position $z$ on its momentum according to

$$
\Delta z=\eta \delta
$$
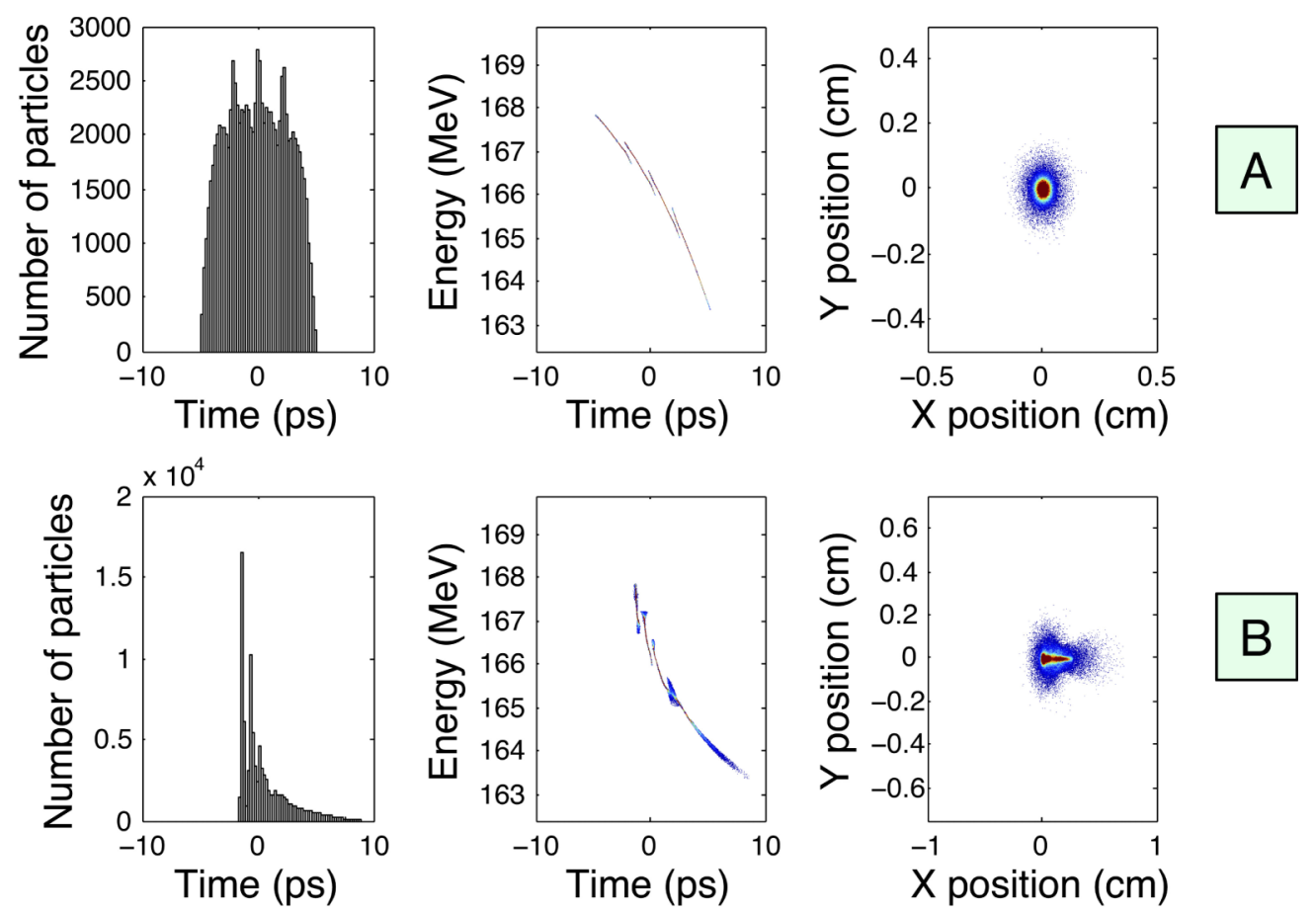

FIG. 6. Compression of the four-pulses comb by magnetic chicane. The third RF cavity $S 3$ is $18^{\circ}$ off crest and $R_{56}=-60 \mathrm{~mm}$ for the chicane. (a) Longitudinal profile, longitudinal phase-space, and transverse spot of the four-pulses comb at the end of the accelerating sections. (b) Longitudinal profile, longitudinal phase-space, and transverse spot of the comb at the CTR target (second THz station). 


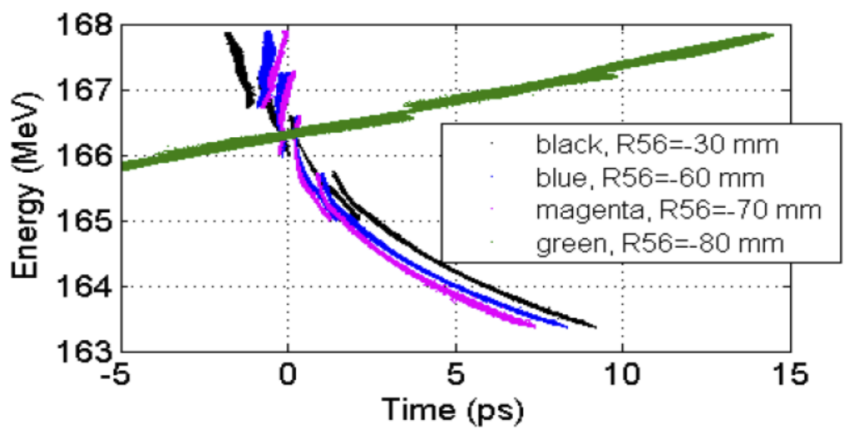

FIG. 7. Compression scan of the four-pulses comb by magnetic chicane. The phase of the third accelerating cavity $S 3$ is fixed at $18^{\circ}$, while the $R_{56}$ of the chicane is scanned around the maximum compression point of the train.

where $\delta=\frac{\Delta E}{E}$ is the relative energy deviation of the electron from the reference particle's energy.

The sign of the momentum compaction in the chicane determines whether the higher energy particles do a longer or a shorter path in it. The energy chirp has to be set accordingly in order to have a rotation of the longitudinal phase space which leads to a shorter electron bunch length.

We can expand $\Delta z$ according to

$$
\Delta z=\eta \delta \approx R_{56} \delta+T_{566} \delta^{2}+U_{5666} \delta^{3},
$$

where the linear longitudinal dispersion is called $R_{56}$, the second order longitudinal dispersion is called $T_{566}$, and the third order one is named $U_{5666 .}{ }^{28}$

In the SPARC case, a chicane having a negative $R_{56}$ has been designed. The chicane is able to compress the single subbunches having the typical energy chirp produced by the space charge (i.e., higher energy in the head of the sub-bunch) and it was initially thought to be operated in combination with an on-crest train. ${ }^{29}$ Later on, we have found out that a higher peak current in the first bunches of the train can be achieved by running the train off crest in one of the accelerating cavities. The latter method allows also to tune better than the distance between the sub-bunches. In this case, the train is injected into a RF cavity with a positive phase (thus with an opposite field slope with respect to the velocity bunching case). With this compression setup, the non-linearity induced by the RF curvature and the bunch compressor partially cancel each other out. The opposite configuration (negative off-crest phase in the RF cavity and negative $R_{56}$ in the chicane) has been rejected because of the unfavourable sum of the RF and magnetic nonlinearity.

In Figure 6(b), an example of such a compression for the same input comb distribution previously discussed is plotted. Both the remaining RF non-linearity and higher orders of the longitudinal dispersion $T_{566}$ and $U_{5666}$ of the dogleg and the chicane give rise to the curvature in the longitudinal phase space shown in Fig. 6(a). In this case, it is not possible to work in an over-compression setup, because the structure of the subbunches is lost after crossing the maximum compression of the train, as shown in Fig. 7.

In the magnetic compression case, the maximum compression point of the sub-bunches is relatively close to the point of maximum compression of the train; therefore, the
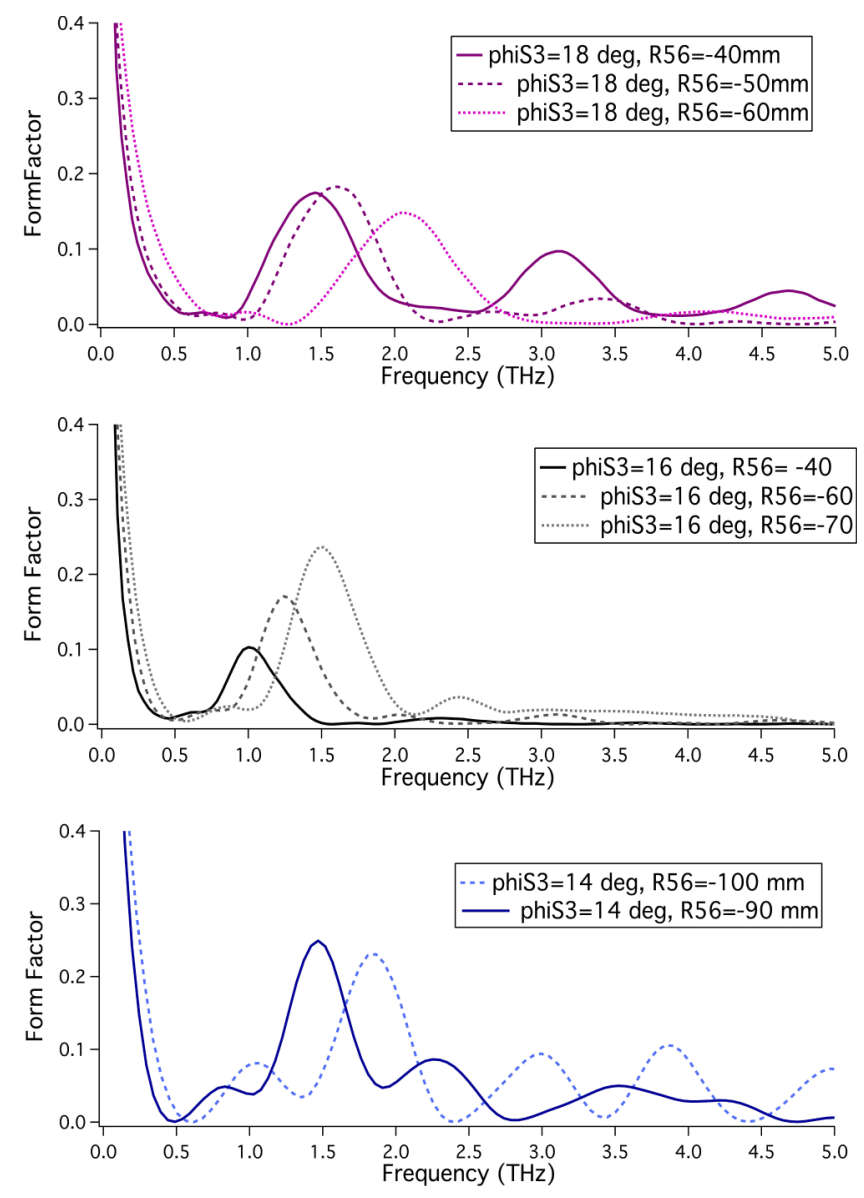

FIG. 8. Form factors corresponding to the 4-pulses comb distribution at the second THz station. Different magnetic compression settings are compared: the $R_{56}$ of the magnetic compressor and the phase of the third accelerating cavity have been varied. The first two accelerating cavities were operated on crest.

final energy spread of the comb is higher than for the velocity bunching case.

The form factor corresponding to Fig. 6(b) is shown in Fig. 8(a) (light pink curve). Different combinations of energy chirp and $R_{56}$ can be used to tune the resonance frequency.

\section{Correction scheme by using an X-band cavity}

The main limit arising from the presented schemes of compression is represented by the difficulty of obtaining a fully periodic train. We have already discussed that this limitation is a consequence of the non-linear terms present in the expansion of the RF voltage amplitude $V$ versus the injection phase $\phi$. This effect can be compensated by the addition of a higher order harmonic RF cavity after the gun.

A linearizing RF cavity is a standard tool that is used in many facilities worldwide. It is typically used either to reduce the final bunch length of the beam or to make the shape of the bunch current more symmetrical. We propose here another use of this well-known tool to preserve the spacing of an initially periodical charge distribution.

We also need to stress that in the configuration that we are going to describe, the linearizer works together with the velocity bunching technique, as first proposed in Ref. 24, which is a scheme not yet in use in any other laboratory. 

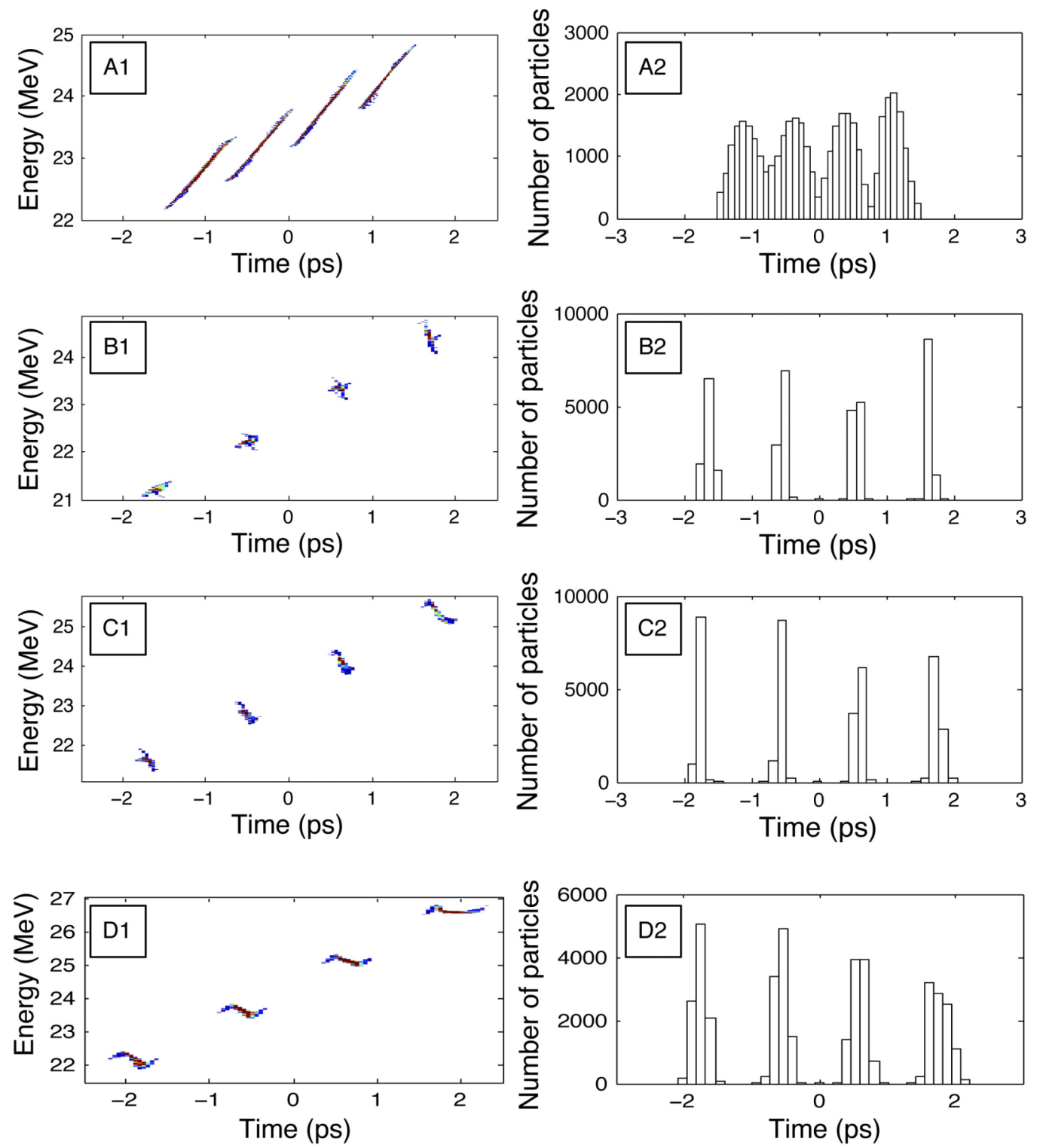

FIG. 9. Longitudinal phase space and charge distribution of a 4-sub-pulses comb beam at the exit of the RF compressor $S 1$ for different over-compression phases and correction of non-linearity by the fourth harmonic cavity. The injection phase in S1 is (a) $-94^{\circ}$, (b) $-98^{\circ}$, (c) $-100^{\circ}$, and (d) $-103^{\circ}$.

The second order term in Eq. (3) can be canceled by using a cavity with frequency $h f$, where $h$ is the harmonic number, such that

$V_{h}(\phi) \approx V_{h} \sin \left(\phi_{h}\right)+h \Delta \phi V_{h} \cos \phi_{h}-\frac{1}{2}(h \Delta \phi)^{2} V_{h} \sin \phi_{h}$

It is important to mention that this formula gives only a preliminary estimation of the necessary correction to be applied; the longitudinal dynamic is indeed more complex due to the RF slippage in the RF compressor and the space charge effect, as described in Ref. 30. Anyhow, the equation delivers a good starting point that can then be better optimized by using numerical codes.

The hypothesis of the insertion of an X-band structure immediately after the gun in the SPARC linac has already been investigated in the past for the single bunch compression; for this reason, a compact standing wave accelerating structure operating at a frequency $\mathrm{f}=11.424 \mathrm{GHz}$, with $5 \mathrm{MeV}$ energy gain, has already been designed. ${ }^{31,32}$ In Ref. 33, the optimiza- tion of the RF compression for the SPARC nominal bunch has been analyzed in details.

Figures 9 and 10 present the results obtained by placing the X-band cavity between the gun and the first acceleration cavity (as shown in Fig. 2).

The cancellation of the second order term in Eq. (3) due to the one in Eq. (6) has the consequence that the distance between two subsequent bunches after their compression is the same within the entire train (see Figure 9).

If we set the phase of the RF compressor to $-100^{\circ}$ (as in plot (c1) in Fig. 9), the bunches have their maximum compression at the exit of the linac but they become longer during the transit in the dogleg. If we set the phase of the RF compressor to $-103^{\circ}$ (as in plot (d1)), the bunches reach their maximum compression at the exit of the dogleg, close to the CTR target, thus the CTR emission is maximized (see plot (c) in Figure 10).

In Figure 10, we present the form factors corresponding to the simulated trains. In plot (b), it can be noticed that the 

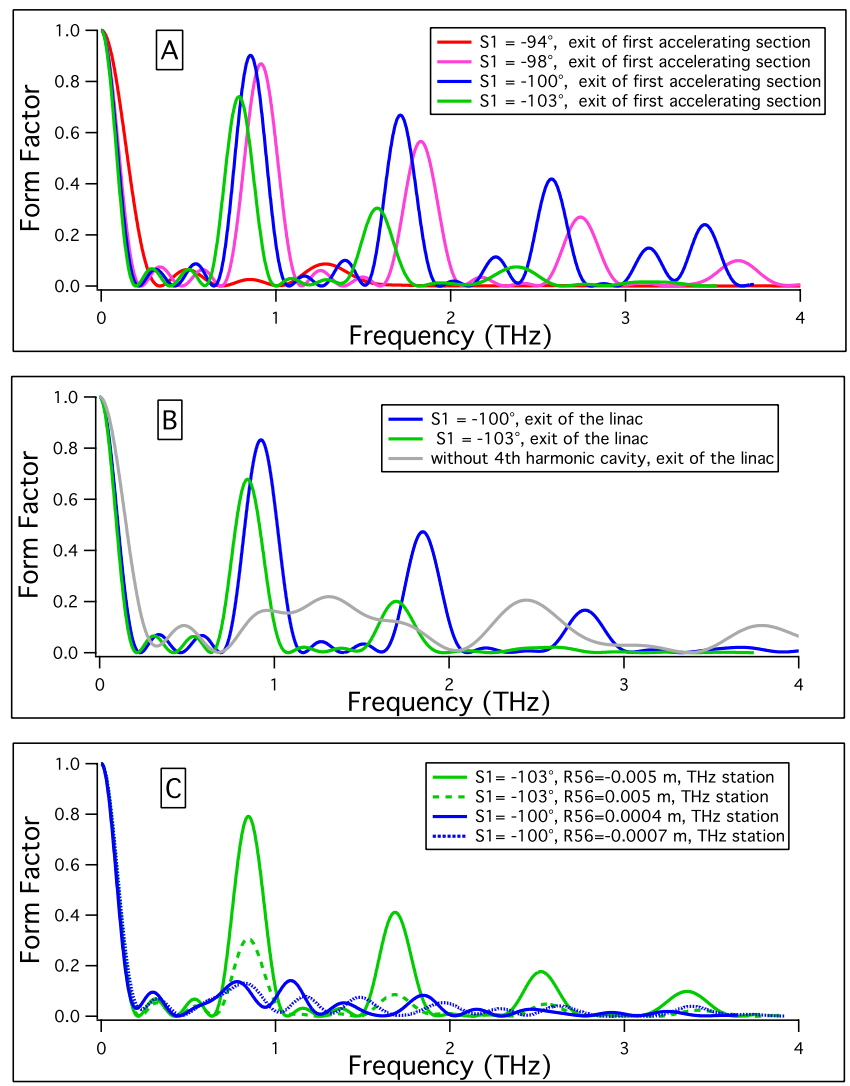

FIG. 10. Form factors of the comb beam for different RF phases, including the correction of non-linearity done by the fourth harmonic cavity. (a) Form factors at the exit of the RF compressor corresponding to the four distributions in Fig. 9, (b) form factors at the exit of the linac compared with one typical form factor obtained without the use of the 4th harmonic cavity, and (c) form factors at the second $\mathrm{THz}$ station for different $R_{56}$ values in the dogleg.

height of the peak at about $1 \mathrm{THz}$ has been more than double with respect to the case without the non-linearity correction. Moreover, its bandwidth is significantly narrower than the optimized case without the X-band insertion. We see also that the second and third peaks deliver a non-negligible radiation intensity.

\section{Considerations about the wakefields impact}

Longitudinal RF wakefields typically scale as the square of the RF frequency, while the transverse wakes scale as the third power of the RF frequency. ${ }^{34}$ Since we are considering to employ a cavity which works at high frequency $(11.424 \mathrm{GHz})$, we would like to discuss here the impact of the short range RF wakefields on the beam dynamics.

The code $\mathrm{ECHO}^{35}$ was used to estimate the wake functions of the X-band cavity by using the method discussed in Ref. 36. The geometry described in Ref. 32 has been used for the calculation. The longitudinal wake function of the X-band cavity reads

$$
W_{0 L}=-\theta(s)\left[293 e^{-\sqrt{z / 0.0003}}-0.89 \frac{\cos \left(47 s^{0.035}\right)}{\sqrt{s}+1.5 s^{-0.89}}\right],
$$

and Figure 11 shows the impact of such a wake on the beam dynamics of an illustrative $200 \mathrm{pC}$ train of bunches tracked
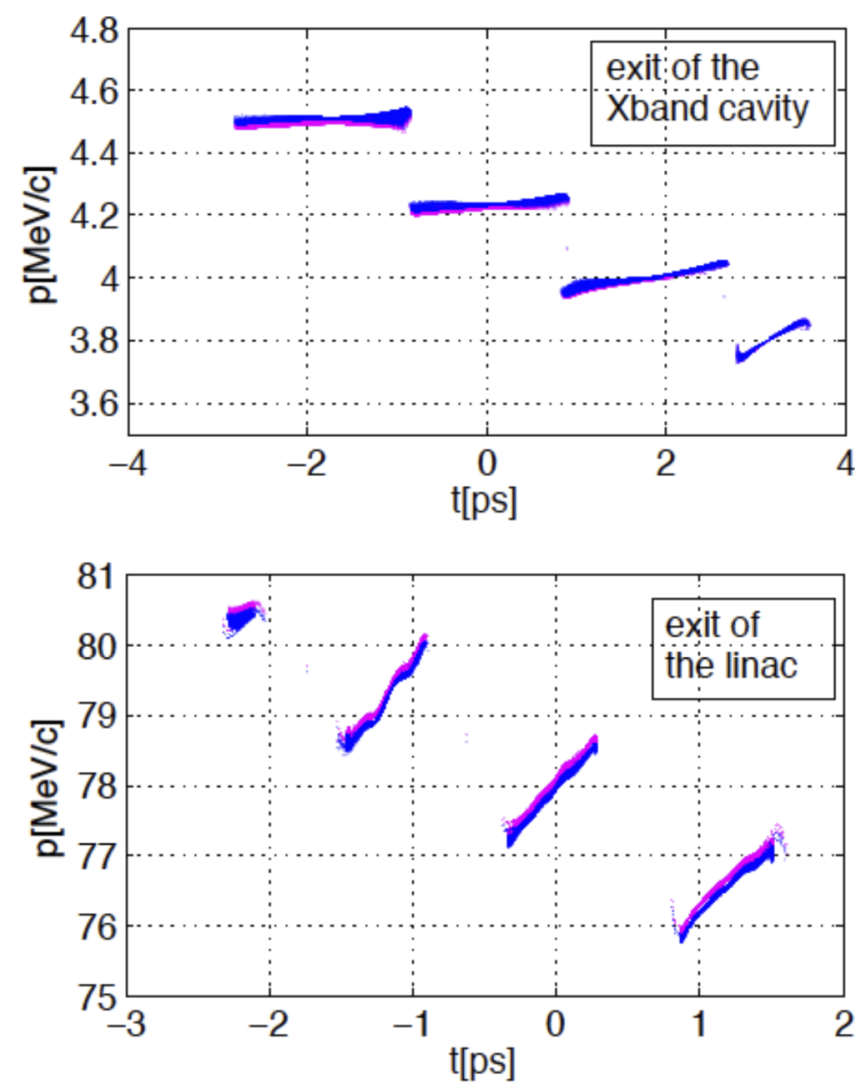

FIG. 11. Top: Comparison between the longitudinal phase space of a train of pulses with (blue points) and without (pink points) the inclusion of the longitudinal wakefield related to the transit in the X-band cavity after the $\mathrm{X}$-band cavity. Bottom: Same comparison at the exit of the linac.

and compressed by using the code ASTRA. ${ }^{37}$ It can be easily seen that the effect of the longitudinal wake is negligible.

On the other side, transverse wakefields are excited in the beam traveling off axis through an accelerating structure. The fields excited by the head of the bunch kick its tail even more off axis: the beam develops a "banana-like" shape and its emittance is spoiled. ${ }^{38}$ There are two main sources of offset: the misalignment of the RF cavity and the beam jitter or beam offset. From the practical experience, the beam transverse jitter is within few tens of micrometers; therefore, in the following, we discuss the effect of the $\mathrm{X}$-band cavity misalignment on the transverse emittance, assuming the beam jitter negligibly small.

The HomDyn code, ${ }^{39}$ which uses the multi-slice approximation to include off-axis beam dynamics, has been used to investigate the emittance growth as a function of the X-band cavity misalignment. The result of this study is reported in Figure 12. We consider the maximum increase of a factor 2 of the beam emittance as acceptable; therefore, the alignment between the beam and the cavity must be better than $0.5 \mathrm{~mm}$.

\section{Summary and comparison among the different schemes}

In this section, we have applied different compression methods to a comb beam. We have examined 3 cases:

1. pure RF compression;

2. pure magnetic compression; 


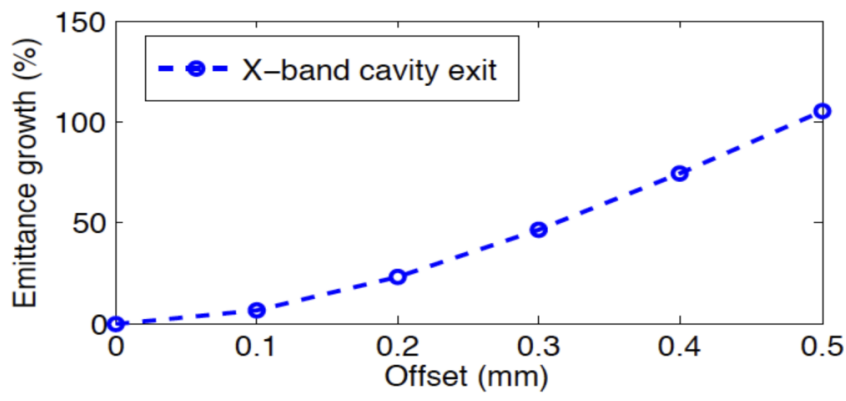

FIG. 12. Emittance degradation in percent as a function of the offset from the nominal axis at the exit of the X-band cavity.

3. RF compression including the compensation of the nonlinear terms in the longitudinal phase space of the comb beam through the insertion of an X-band cavity after the gun.

Pure RF compression scheme (1) has been already used at SPARC and has produced the first experimental results. The main limitation of this scheme is given by the fact that the bunch train looses its periodicity after its compression due to the RF non-linearity. In Sec. II A we have shown that it is possible to produce with this scheme intense, narrow bandwidth $\mathrm{THz}$ radiation at tunable frequencies. Specifically, the obtained form factors have the first peak with amplitude in the range 0.2-0.4 and position shiftable between 0.7 and $1.4 \mathrm{THz}$.

Pure magnetic compression scheme (2) does not present any big advantage with respect to the actual setup. Indeed, we have shown in Sec. II B that neither higher intensity nor narrower bandwidth with respect to the velocity bunching case could be obtained. It must be also recalled that the presented simulations neglect the CSR effect that might further spoil the electron bunch quality.

The main limit of this setup for the application as $\mathrm{THz}$ source is caused by the fact that is more difficult to separate the maximum compression point of the sub-bunches from the maximum compression point of the overall train, preventing the production of four short, well separated bunches, with low total energy spread.

Scheme number (3) turned out to be the best setup. We have indeed shown that the intensity of the $\mathrm{THz}$ radiation at the fundamental frequency is more than doubled, its bandwidth is reduced and higher order harmonics become non-negligible (as shown in Figure 10). The presence of strong wakefields due to the small iris of the cavity and their impact could be reduced by a precise alignment of the beam trajectory. More specifically, an alignment between the beam and the cavity better than $0.5 \mathrm{~mm}$ has to be reached. The smallness of the X-band iris has an other drawback: in order to focus the beam at its entrance, the current of the main solenoid can be adjusted only within a small range, reducing the tuning space for the transverse emittance minimization. Nevertheless, as we already mentioned, the CTR emission does not have strong requirements on the beam emittance.

For the sake of completeness, we have to mention that in this review, we have not dealt with the discussion of a possible scheme including magnetic compression used in combination with a RF linearizing cavity. Nevertheless, references concern- ing this setup can be easily found in the literature because it is already in use in facilities like SLAC and FLASH. ${ }^{40,41}$

\section{CONCLUSIONS}

In this paper, we have shown how it is possible to tune the wavelength of the narrow band $\mathrm{THz}$ source at $S P A R C_{-} L A B$ by changing the machine parameters. We have compared the results provided by different compression techniques in use at $S P A R C_{2} L A B$ and we have highlighted the present limits for the compression of the electron bunch train. We have shown through simulations that a huge improvement in the power of the coherent transition radiation emitted by the SPARC comb beam could be obtained by the insertion of an X-band cavity at the gun exit.

${ }^{1}$ Y. Shen et al., Phys. Rev. Lett. 107, 204801 (2011).

${ }^{2}$ C. Ronsivalle et al., New J. Phys. 16, 033018 (2014).

${ }^{3}$ V. Petrillo et al., Phys. Rev. Lett. 111, 114802 (2013).

${ }^{4}$ A. Marinelli et al., Nat. Commun. 6, 6369 (2015).

${ }^{5}$ E. Allaria et al., Nat. Commun. 4, 2476 (2013).

${ }^{6} \mathrm{M}$. Litos et al., Nature 515, 92-95 (2014).

${ }^{7}$ P. Muggli et al., Phys. Rev. Spec. Top.-Accel. Beams 13, 052803 (2010).

${ }^{8}$ P. Piot et al., Appl. Phys. Lett. 98, 261501 (2011).

${ }^{9}$ Y. Kim et al., in Proceedings of PAC13, MOPH020, Pasadena, CA, U.S.A., 2013.

${ }^{10} \mathrm{R}$. Agustsson et al., in Proceedings of IPAC13, WEPWA080, Shanghai, China, 2013.

${ }^{11}$ A. Zholents and M. Zolotev, Phys. Rev. Lett. 76, 912 (1996).

${ }^{12}$ J. G. Neumann et al., J. Appl. Phys. 105, 053304 (2009).

${ }^{13}$ S. Antipov et al., Phys. Rev. Lett. 111, 134802 (2013).

${ }^{14} \mathrm{M}$. Ferrario et al., Nucl. Instrum. Methods Phys. Res., Sect. B 309, 183-188 (2013).

${ }^{15}$ F. Villa et al., Nucl. Instrum. Methods Phys. Res., Sect. A 740, 188-192 (2014).

${ }^{16}$ A. Mostacci et al., in Proceedings of IPAC11, THYB01, San Sebastian, Spain, 2011.

${ }^{17}$ M. Boscolo et al., Nucl. Instrum. Methods Phys. Res., Sect. A 577, 409-416 (2007).

${ }^{18}$ M. Ferrario et al., Nucl. Instrum. Methods Phys. Res., Sect. A 637, S43-S46 (2011).

${ }^{19}$ E. Chiadroni et al., Rev. Sci. Instrum. 84, 022703 (2013).

${ }^{20}$ E. Chiadroni et al., Appl. Phys. Lett. 102, 094101 (2013).

${ }^{21}$ T. Levato et al., Nucl. Instrum. Methods Phys. Res., Sect. A 720, 95-99 (2013).

${ }^{22} \mathrm{C}$. Vaccarezza et al., in Proceedings of IPAC14, MOPRO078, Dresden, Germany, 2014.

${ }^{23}$ L. M. Young, "TSTEP is a derivative of PARMELA code," PARMELA Report No. LA-UR-96-1835 (Los Alamos National Laboratory, 1996 (revised 2005)).

${ }^{24}$ L. Serafini and M. Ferrario, AIP Conf. Proc. 581, 87 (2001).

${ }^{25}$ M. Ferrario et al., Phys. Rev. Lett. 104, 054801 (2010).

${ }^{26} \mathrm{~B}$. Marchetti, "Development of a high energy tunable $\mathrm{THz}$ source at SPARC," Ph.D. thesis, 2012; available at http://www.infn.it/thesis/thesis dettaglio.php?tid=6347.

${ }^{27}$ B. Marchetti et al., in Proceedings of IPAC11, THPS101, San Sebastian, Spain, 2011.

${ }^{28}$ Y. Sun et al., SLAC-PUB-14327 (Stanford Linear Accelerator Center, SLAC-PUB Department, Stanford, CA 94309, U.S.A., 2011).

${ }^{29}$ M. Boscolo et al., in Proceedings of EPAC, WEPC069, Genoa, Italy, 2008.

${ }^{30}$ A. Bacci and A. R. Rossi, Nucl. Instrum. Methods Phys. Res., Sect. A 740, 42-47 (2014).

${ }^{31}$ D. Alesini et al., SPARC-RF-03/002 (INFN LNF, Via Enrico Fermi, 40, 00044 Frascati RM, Italia, 2003).

${ }^{32}$ A. Bacci et al., SPARC-RF-03/001 (INFN LNF, Via Enrico Fermi, 40, 00044 Frascati RM, Italia, 2003).

${ }^{33}$ C. Ronsivalle et al., SPARC-BD-05/01 (INFN LNF, Via Enrico Fermi, 40, 00044 Frascati RM, Italia, 2005). 
${ }^{34} \mathrm{~W}$. Barletta, Unit 4 - Lecture 13 - Beam Loading and Wakefields, Lecture at the US Particle School, 2012.

${ }^{35}$ I. Zagorodnov and T. Weiland, Phys. Rev. Spec. Top.-Accel. Beams 8, 042001 (2005).

${ }^{36}$ I. Zagorodnov et al., TESLA Report 2004-01 (2004).

${ }^{37}$ K. Floettmann, ASTRA, A Space Charge Tracking Algorithm, 2011, DESY, Notkestr.85, 22603 Hamburg, Germany.

${ }^{38}$ M. Ferrario et al., Int. J. Mod. Phys. A 22(23), 4214-4234 (2007).
${ }^{39}$ M. Ferrario et al., "HOMDYN Study for the LCLS RF Photo-Injector," in The Physics of High Brightness Beams: Proceedings of the 2nd ICFA Advanced Accelerator Workshop, University of California, Los Angeles, USA, 9-12 November 1999 (World Scientific, 2000).

${ }^{40}$ Z. Zhang et al., Phys. Rev. Spec. Top.-Accel. Beams 18, 030702 (2015).

${ }^{41} \mathrm{C}$. Entrena, "Erzeugung und transport von doppelpaket-elektronenstrahlen im FLASH linearbeschleuniger," DESY-2014-03169, M.S. thesis, DESY, Notkestr.85, 22603 Hamburg, Germany, 2014. 